\title{
Analysis of the dust evolution in the circumstellar disks of T Tauri stars
}

\author{
A. Schegerer ${ }^{1}$, S. Wolf ${ }^{1}$, N. V. Voshchinnikov ${ }^{2,3}$, F. Przygodda ${ }^{1}$, and J. E. Kessler-Silacci ${ }^{4}$ \\ 1 Max Planck Institute for Astronomy, Königstuhl 17, 69117 Heidelberg, Germany \\ e-mail: schegerer@mpia.de \\ 2 Sobolev Astronomical Institute, St. Petersburg University, Universitetskii prosp. 28, St. Petersburg 198504, Russia \\ Isaac Newton Institute of Chile in Eastern Europe and Eurasia, St. Petersburg Branch, Russia \\ ${ }^{4}$ Department of Astronomy, University of Texas, C-1400, Austin, TX 78712, USA
}

Received 21 November 2005 / Accepted 22 May 2006

\section{ABSTRACT}

\begin{abstract}
Aims. We present a compositional analysis of $8-13 \mu \mathrm{m}$ spectra of 32 young stellar objects (YSOs). Our sample consists of 5 intermediate-mass stars and 27 low-mass stars. Although some previous studies give reasons for the similarity between the dust in circumstellar disks of T Tauri stars and Herbig Ae/Be stars, a quantitative comparison has been lacking so far. Therefore, we include a discussion of the results of the $10 \mu \mathrm{m}$ spectroscopic survey of van Boekel et al. (2005, A\&A, 437, 189), who focus on Herbig $\mathrm{Ae} / \mathrm{Be}$ stars, the higher mass counterparts of T Tauri stars, and draw comparisons to this and other studies.

Methods. While the spectra of our 32 objects and first scientific results have already been published elsewhere we perform a more detailed analysis of the $\sim 10 \mu \mathrm{m}$ silicate feature. In our analysis we assume that this emission feature can be represented by a linear superposition of the wavelength-dependent opacity $\kappa_{\mathrm{abs}}(\lambda)$ describing the optical properties of silicate grains with different chemical composition, structure, and grain size. Determining an adequate fitting equation is another goal of this study. Using a restricted number of fitting parameters, we investigate which silicate species are necessary for the compositional fitting. Particles, with radii of $0.1 \mu \mathrm{m}$ - and $1.5 \mu \mathrm{m}$ and consisting of amorphous olivine and pyroxene, forsterite, enstatite, and quartz are considered. Only compact, homogeneous dust grains are used in the presented fitting procedures. In this context we show that acceptable fitting results can also be achieved if emission properties of porous silicate grains are considered instead.

Results. Our analysis shows - in terms of the properties of the circumstellar dust-like crystallinity - T Tauri systems are a continuation of HAeBe systems at their lower mass end. However, a weak correlation between grain growth and stellar luminosity could be found, in contrast to HAeBe systems.
\end{abstract}

Key words. astrochemistry - stars: pre-main sequence - infrared: stars - lines: profiles

\section{Introduction}

T Tauri stars are known as precursors of low-mass main sequence stars similar to our sun. In contrast to their more evolved counterparts, one of their characteristics is excess radiation observed in the infrared and sub-millimeter range. Highresolution interferometric observations (e.g., Leinert et al. 2004; van Boekel et al. 2004; Millan-Gabet et al. 2001), combined with radiative transfer models (e.g., Cotera et al. 2001; Wolf 2003c; Akeson et al. 2005), confirm what has been assumed for 30 years (Mendoza 1968; Adams et al. 1987): the excess arises from a circumstellar, passive disk heated by the central star. Last, but not least, images in the near-infrared up to the sub-millimeter wavelength regime have provided impressive illustrations of the circumstellar structure of young stellar objects (YSOs) (e.g., Padgett et al. 1999; Allen et al. 2002; Krist et al. 2005).

Several different absorption and emission features have been detected towards YSOs so far (e.g., Molster \& Waters 2003 and Table 1 in Voshchinnikov 2002). A broad, frequently-found emission band is the silicate feature at $\sim 10 \mu \mathrm{m}$ that is attributed to the stretching mode of the $\mathrm{Si}-\mathrm{O}$ bond in silicate minerals (e.g., Dorschner et al. 1988). Hanner et al. (1995) successfully reproduced this feature and the underlying continuum by a two component fit: $\lambda \mathcal{F}_{\lambda} \propto C_{0} \lambda^{n}+C_{1} \varepsilon(\lambda) \lambda^{m}$, where $C_{0}$ and $C_{1}$, as well as $m$ and $n$, are fitting constants, and $\varepsilon(\lambda)$ is the normalized, smoothed silicate band of $\theta$ Ori D (Elias 1978) in the $N$ band ${ }^{1}$. The first term represents the underlying continuum from the optically thick, inner region of the disk, while the second term stands for the emission feature, which is assumed to have its origin in the optically thin surface layer (Natta et al. 2000). Instead of using the normalized emission feature of an arbitrarily selected source, Bouwman et al. (2001) performed compositional fits that are based on the emission profiles of different silicate compounds, particle shapes, and radii $(0.1 \mu \mathrm{m}$ and $2.0 \mu \mathrm{m}$ for olivine, $0.1 \mu \mathrm{m}$ for forsterite, enstatite, and quartz). This approach was motivated by previous studies of the optical parameters of different silicate minerals (e.g., Dorschner et al. 1995). The resulting profile is a linear combination of the opacities $\kappa_{\mathrm{abs} ; i}(\lambda)$ for the different silicate species $i$.

As for the observed shift in peak position of the silicate band, Bouwman et al. (2001) found a change in average grain size and dust crystallisation in Herbig $\mathrm{Ae} / \mathrm{Be}(\mathrm{HAeBe})$ stars indicating dust-grain evolution. In addition, this result was supported both by further comparisons with mid-infrared spectra of the interstellar medium as the parent material for YSOs, and the midinfrared spectra of the comet Hale Bopp as a representative of the evolved planetary systems. While the mid-infrared flux of

\footnotetext{
${ }^{1} N$ band covers the wavelength range $8.0-13.0 \mu \mathrm{m}$.
} 
interstellar medium shows a maximum at a wavelength of $\sim 9.8 \mu \mathrm{m}$, the silicate feature of comet Hale Bopp is flatter with a maximum at $\sim 11.3 \mu \mathrm{m}$. Corresponding results were found in laboratory for silicate grains of increasing size and crystallinity.

Because of instrumental sensitivity constraints, most studies have focused on HAeBe stars rather than on the fainter T Tauri systems up to the present. Apart from the similarity of general properties like geometry and structure, evolutionary processes like dust coagulation and crystallization are assumed to be accelerated or at least enabled in HAeBe stars because of the higher energy input from the star. There are only a few authors who analysed the $10 \mu \mathrm{m}$-feature of classical T Tauri stars, so far. Natta et al. (2000) presented low-resolution spectra of nine classical $\mathrm{T}$ Tauri stars associated with the Chameleon I dark cloud. Honda et al. (2003) found a larger amount of crystalline silicate like forsterite, enstatite and quartz in the primary of the well-studied binary Hen 3-600. Furthermore, Przygodda et al. (2003, hereafter PR03) and Kessler-Silacci et al. (2005, hereafter KS05) selected a sample of $16 \mathrm{~T}$ Tauri and $5 \mathrm{HAeBe}$ stars. They established a linear correlation between feature strength and feature shape which were explained by grain growth. The similar correlation has been ascertained for HAeBe stars (van Boekel et al. 2003, 2005).

In this paper we complete the work presented in PR03 and KS05 by providing a more detailed analysis of the silicate emission feature. While the objects are introduced in Sect. 2, in Sect. 3 the fitting function is scrutinised in detail in respect of the temperature (Sect. 3.1) and the emission properties (Sect. 3.2) of the dust grains which contribute to the silicate feature. After studying our fit results (Sect. 4) we investigate the dependence of grain growth and crystallization on stellar parameters and look for further correlations. Finally, we raise the question, if the silicate feature could be reproduced by dust species that have not been considered, so far (Sect. 5). In this context, porous dust grains are taken into account. A discussion of future prospects conclude this study in Sect. 6.

\section{The sample}

Our sample of 27 T Tauri is presented in Table 1. We additionally consider $5 \mathrm{HAeBe}$ stars in order to show differences and similarities between low-mass and intermediate-mass systems. Furthermore, we are able to analyse differences between our results and previous studies. The targets were observed in the $N$ band with the Thermal Infrared Multi Mode Instrument 2 (TIMMI2; Reimann et al. 1998, 2000) at ESO's observatory La Silla and with the Long Wavelength Spectrometer (LWS) at the W. M. Keck Observatory (e.g., Marler et al. 1995). Information about the observations and the subsequent data reduction are given in PR03 and KS05.

Six of our targets are known binaries (e.g., VWCha, Hen 3-600 A, AK Sco), but we have seperate spectra only for two binary objects (AS 205 and S CrA). Therefore, we present all of the spectral analysis of $(30+2)$ objects together. Selection effects in terms of stellar age and stellar mass cannot be found (see Table 1), although most of our objects are younger (age $<3 \mathrm{Myr}$ ) T Tauri stars. Assuming similar stellar masses, the putative more frequent observation of younger T Tauri stars can be explained by their stronger infrared brightness in contrast to older T Tauri stars (>6 Myr), where the circumstellar disk, which is responsible for the infrared excess, has already vanished (Haisch et al. 2001; Carpenter et al. 2005). The age is certainly the least constrained parameter in Table 1.
Considering the inclination of the circumstellar disks of the objects as a further, geometrical selection effect, the appearance of an emission feature favours smaller inclination angles ${ }^{2}$ if the feature originates from the innermost regions of the optically thin surface of the disk.

\section{The fitting function}

The determination of an adequate fitting function of the $10 \mu \mathrm{m}$ silicate feature and its physical justification is non-trivial. The difficulty results from trying to simulate the complex spectral energy distribution (SED) with the least number of parameters.

In fact, our first approach was to determine of a potential grain-size distribution of dust in a circumstellar disk by only considering the $10 \mu \mathrm{m}$ silicate feature. This approach failed because unique fitting models could not be obtained. In this context, we have to mention that Mathis et al. (1977) found a particle size distribution for graphite and several silicates in interstellar dust that can roughly be reproduced by a power law. It is still an open issue if a similar particle size distribution exists in the environment of YSOs, too (e.g., Tanaka et al. 1996).

Nevertheless, it has been found that a simple function with only a small number of fitting parameters finally guarantees a successful, robust fit. Therefore, the balance between physical justification and fitting robustness is our guiding idea.

In order to compare our results with previous ones, we consider an ansatz, which Hanner et al. (1995) and BO05 used:

$\mathcal{F}_{v}=B_{v}(T)\left(C_{0}+\sum_{i=1}^{n} C_{i} \kappa_{\mathrm{abs} ; i}\right)$,

where $C_{0}$ and $C_{i}$ are the fitting parameters, $\kappa_{\mathrm{abs} ; i}$ represents the wavelength-dependent opacity for dust component $i$, and $B_{v}(T)$ is the Planck function corresponding to temperature $T$ (see Eq. (2)). In Sects. 3.1 and 3.2 we include more detailed discussions of Eq. (1).

Two fitting procedures are considered. First, we examine a fitting procedure that is based on an iterative LevenbergMarquardt least-square minimization (Press et al. 1986). But, this numerical fitting method does not guarantee the best fit, i.e. the finding of the global minimum of the fit quality parameter $\chi^{2}$ (reduced chi-squared, see Sect. 4), particularly for data with lower signal-to-noise ratios. Repeated modifications of the initial fitting parameters and the subsequent analysis of $\chi^{2}$ allow an effective search for the global minimum of $\chi^{2}$.

Second, we consider the non-negative, linear least-square fit procedure used by Lawson \& Hanson (1974) containing the analytical solution of the matrix equation $\tilde{C}_{i} \tilde{K}_{\mathrm{abs} ; i}=\mathcal{F}_{v}$ with $\tilde{\kappa}_{\mathrm{abs} ; i}=B_{\nu} \kappa_{\mathrm{abs} ; i}$. The matrix equation is solved for each integer temperature value between a minimum and maximum temperature. The temperature with the lowest corresponding $\chi^{2}$ is the temperature $T$ that we look for. We assume a silicate sublimation temperature of $1500 \mathrm{~K}$ (Duschl et al. 1996) and a negligible contribution of the silicate emission to the mid-infrared spectrum if the temperature of the corresponding silicate compound is lower than $50 \mathrm{~K}$ (see Sect. 3.1). Because of robustness and high rapidity the non-negative, linear least-square fit procedure is finally used for our study.

\footnotetext{
${ }^{2}$ With respect of the face-on orientation.
} 


\subsection{The single-temperature black-body approximation}

In Eq. (1) the Planck function has the following form $(x=$ $(h v) /(k T))$ :

$B_{v}(x)=\frac{15}{\pi^{4}} \frac{x^{3}}{\exp (x)-1}$

with Planck constant $h$, Boltzmann constant $k$, and frequency $v$. This function is normalized in such a way that its integral $\int_{0}^{\infty} B_{v}(T) \mathrm{d} v$ is unity.

In fact, dust grains with different temperatures contribute to the SED in the $N$ band. But an extensive study of the silicate feature and the underlying continuum can only be done with radiative transfer simulations in a dust disk model. However, there have been several different efforts to determine the different silicate compounds with simple, semi-analytical tools. Most models are based on the idea that the silicate feature arises from single-temperature dust grains in an optically thin surface layer. Consequently, the emitted flux is given by the formula $\mathcal{F}_{v}=\tau_{v} B_{v}(T)$ with optical depth $\tau_{v}=\sum_{i=1}^{n} C_{i} K_{\text {abs } ; i}$. Although this approach does not consider the dependence of dust temperature on grain size and composition, the mean dust temperature is assumed to be dominated by the absorption and emission characteristics of carbon grains assuming thermal equilibrium (Krügel \& Walmsley 1984). Przygodda (2004) obtained a further simplification of the model by assuming that the temperature of most dust grains, which effectively contribute to the emission feature, is $T \approx 300 \mathrm{~K}$. As a maximum flux $\left[B_{v}(T)\right]_{\max }$ at $\lambda=10 \mu \mathrm{m}$ corresponds to a temperature of $\sim 290 \mathrm{~K}$, the Planck function $B_{v}(T \approx 300 \mathrm{~K})$ is approximately constant in $N$ band and the shape of the feature depends only on the emission properties of dust grains: $\mathcal{F}_{v} \approx \tau_{v}$. In order not to preselect a certain dust temperature, the temperature $T$ can also be introduced as another free-fitting parameter as was done in BO05 and as we do in this study. Also in this approach, the underlying continuum of the $10 \mu \mathrm{m}$-feature is assumed to be dominated by dust grains of the same temperature regime. We have to mention that this approach does not consider the possibility that large reservoirs of cool $(\ll 300 \mathrm{~K})$ and/or hot $(\gg 300 \mathrm{~K})$ dust grains provide the main contribution to the SED at $10 \mu \mathrm{m}$. For this reason further analysis requires the modeling of a more representative circumstellar disk. Such a representative model of a circumstellar disk is generated by means of a Monte Carlo code for radiative transfer simulations (MC3D: Wolf et al. 1999; Wolf 2003a,b). The density distribution is calculated assuming hydrostatic equilibrium in the vertical direction, i.e. perpendicular to the disk midplane (Schegerer et al., in preparation).

For this study, stellar properties are chosen that were recently found by modeling the SED of the low-mass star RY Tau (Schegerer et al., in prep.; Akeson et al. 2005). The inner and outer radii of the disk amount to $0.3 \mathrm{AU}$ and $100 \mathrm{AU}$, respectively. As absorbing, scattering, and emitting material, we use a mixture of carbon and silicate dust in a 1:2 ratio (Weingartner \& Draine 2001; Draine \& Lee 1984; Mathis et al. 1977) ${ }^{3}$. This dust mixture has been used in a large variety of simulations of circumstellar dust configurations (e.g., Wolf et al. 2003). The extinction, absorption, and scattering cross sections are averaged over their abundance. Such an approach minimises the required computer power and memory as the temperature and density distribution does not have to be determined and stored for each dust type (Wolf 2003c). Figure 1 shows spectra that are obtained from systems with identical geometry and identical stellar properties,

\footnotetext{
${ }^{3}$ See also http://www . astro.princeton. edu/ draine
}

but in each case only dust grains having a temperature in the indicated temperature interval are taken into account. Although the $10 \mu \mathrm{m}$ flux is maximised for a temperature $T$ of about $375 \mathrm{~K}^{(4)}$, dust grains with a temperature between $300 \mathrm{~K}$ and $400 \mathrm{~K}$ contribute only moderately ( $20 \%)$ to the spectra compared with grains in adjacent temperature intervals. Therefore, the $N$ band of the SED cannot be assigned to a certain temperature interval or a certain area of the disk. In reality, all grains with different temperatures contribute to this feature.

However, the temperature that we obtain in our singletemperature approximation is an average temperature of all dust grains weighted by their spectral contribution to the observed silicate feature. The average itself is assured by the monotonic gradient of the spectral contribution of dust grains with temperatures in the interval between $100 \mathrm{~K}$ and $600 \mathrm{~K}$. Figure 2 shows the fitting quality parameter $\chi^{2}$ (see Eq. (3)) of some fits versus the pre-specified temperature $T$ for the Planck function (2). The existence of a global minimum of $(\chi(T))^{2}$ justifies the simplifying single-temperature-model of the underlying spectral continuum in $N$ band.

Future radiative transfer simulations will give the necessary assistance for the decision, if our simplified model for silicate emission in a circumstellar disk is physically reasonable. Furthermore, interferometric observation with the mid-infrared interferometer MIDI could help to understand the potential physical meaning of the determined fitting temperature $T$ (Schegerer et al., in prep.).

\subsection{Emission profiles}

The emission profiles that we use for the fitting procedure are calculated for homogeneous compact, spherical grains with the Mie theory starting from the complex refractive indices $n_{i}$ for silicate material $i$ (Dorschner et al. 1995; Servoin \& Piriou 1973; Jäger et al. 1998b; Spitzer \& Kleinmann 1960). The result is the dimensionless absorption efficiency $Q_{\mathrm{abs} ; i}$, which is used for calculating of the opacity ${ }^{5} \kappa_{\mathrm{abs} ; i}=Q_{\mathrm{abs} ; i} \pi r^{2} /\left(4 / 3 \pi r^{3} \rho\right)$, where $r$ is the particle radius and $\rho$ the material density.

Figure 3 shows the opacities for amorphous olivine $\left(\mathrm{MgFeSiO}_{4}\right)$ and pyroxene $\left(\mathrm{MgFe}\left[\mathrm{SiO}_{3}\right]_{2}\right)$, while the lower panels present crystalline forsterite $\left(\mathrm{Mg}_{2} \mathrm{SiO}_{4}\right)$, enstatite $\left(\mathrm{MgSiO}_{3}\right)$, and quartz $\left(\mathrm{SiO}_{2}\right)$, each with radii $0.1 \mu \mathrm{m}, 1.5 \mu \mathrm{m}$, and $2.5 \mu \mathrm{m}$. As Bouwman et al. (2001) point out, one $0.1 \mu \mathrm{m}$ and one $2.0 \mu \mathrm{m}$-sized dust components for each chemical compound seem to be sufficiently representative to model the $10 \mu \mathrm{m}$ silicate-feature. Here, we use dust grains with a radius of $0.1 \mu \mathrm{m}$ and $1.5 \mu \mathrm{m}$ in order to better compare to the results of $\mathrm{BO} 05$. The largest, $2.5 \mu \mathrm{m}$-sized particles show the broad plateau between $9 \mu \mathrm{m}$ and $12 \mu \mathrm{m}$ (see Fig. 3, two upper panels), which does not appear to exist in any of the observations modeled here. The difference in profiles between $1.5 \mu \mathrm{m}$ and $2.5 \mu \mathrm{m}$ sized particles from forsterite, enstatite, and quartz is not significant (Fig. 3, three lower panels). Therefore, the $2.5 \mu \mathrm{m}$ grains are not considered in the fitting procedure. Dust-grains species that do not show an emission feature in the $8-13 \mu \mathrm{m}$ range, such as amorphous, compact carbon grains (e.g., Jäger et al. 1998a), are not considered. However, considering non-homogeneous dust grains (see Sect. 5), which consist of a mixture of various materials, carbon may also modify the silicate emission (see Natta et al. 2001).

\footnotetext{
4 This value is obtained by a more detailed analysis with more narrow temperature intervals.

5 Also known as mass absorption coefficient; e.g., Li (2005).
} 

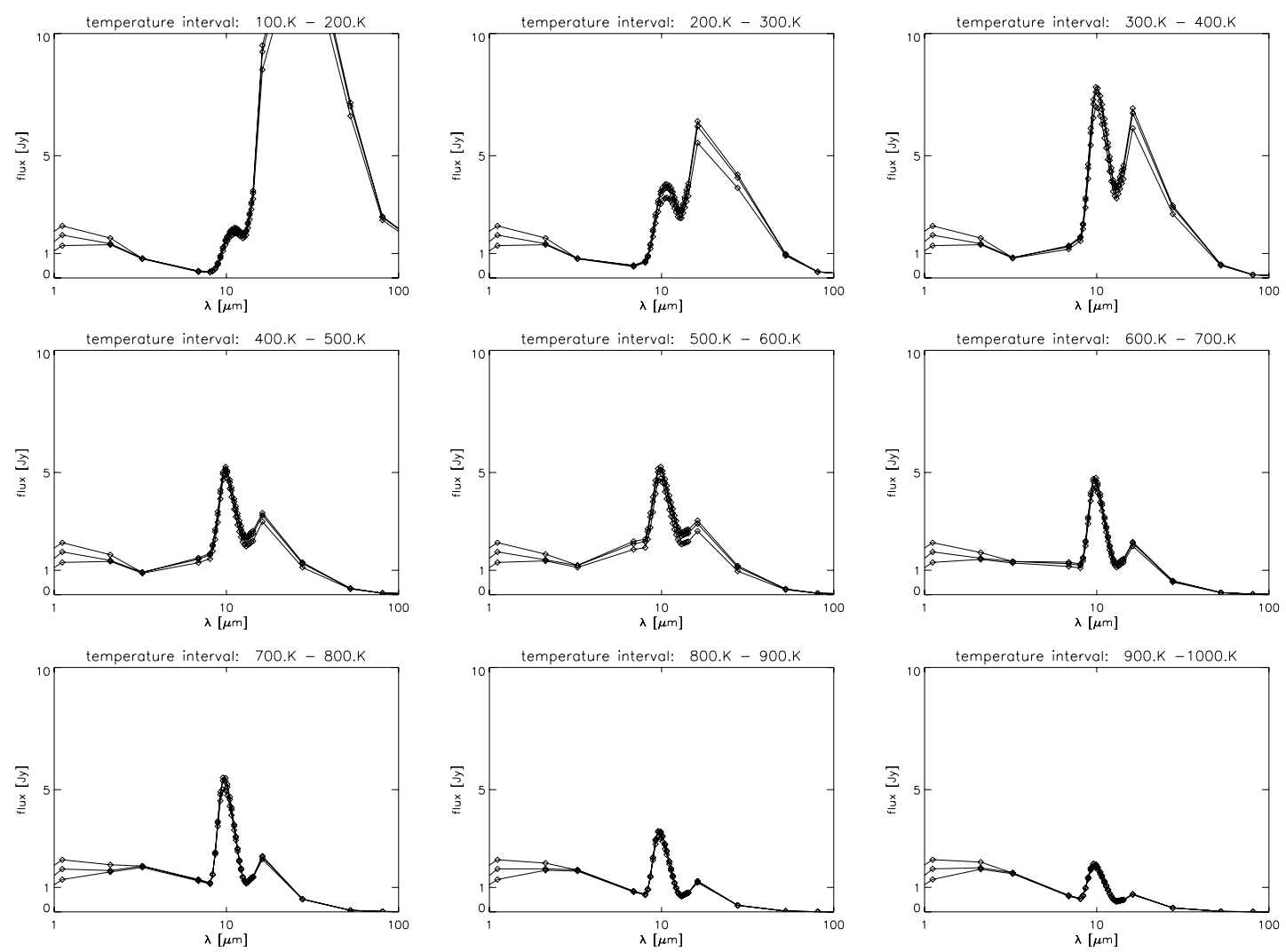

Fig. 1. The simulated SEDs of a T Tauri system assuming a distance of $140 \mathrm{pc}$. The inner and outer radii of the disk amount to $0.3 \mathrm{AU}$ and $100 \mathrm{AU}$, respectively (see Sect. 3.1). The spectra are obtained from systems with identical geometry and identical stellar properties, but in each case only dust grains that have a temperature in the indicated temperature interval contribute to the SED. Each figure shows the SED for inclinations $0^{\circ}$, $15^{\circ}$, and $45^{\circ}$ with respect to face-on orientation. Each SED is calculated for 34 wavelengths $(\diamond)$. The integration of the flux between $5.0 \mu \mathrm{m}$ and $15.0 \mu \mathrm{m}$ yields $1.84,3.9,7.5,5.1,5.5,4.3,4.8,2.8,1.8 \times 10^{14} \mathrm{Jy} \times \mathrm{Hz}$ from the upper left to the lower right figures. The feature at $\sim 20 \mu \mathrm{m}$ is ascribed to silicate, too.

The emission profiles of the remaining dust components are linearly independent of the opacities of the other dust components, as BO05 have shown. This linear independence and the resulting uniqueness of the solution is not ensured if too many emission profiles are included.

Previous investigations (e.g., Natta et al. 2000) do not include certain dust species like enstatite, quartz and polycyclic aromatic hydrocarbons (PAH) in circumstellar disks around lower-mass stars. In general, this assumption does not seem to be justified. Honda et al. (2003) found large mass fractions of enstatite and quartz in the T Tauri object Hen 3-600 A. Finally, the robustness of our fitting procedure allows us to include these dust species with one exception: we do not consider PAHs. The PAH features are strongly variable in peak position and profile from source to source and, therefore, cannot be represented by one unique emission profile. Additionally, there are hints that the PAH features seem to be composed of several subfeatures (Peeters et al. 2002).

\section{Results}

In the following, we focus on the fitting results (see Table 2) and make comparisons to previous publications.

The results of the fitting procedure are generally satisfactory (see Fig. 4). The quality of each fit, characterised by $\chi^{2}$ (e.g., Press et al. 1986) with

$\chi^{2}=\frac{1}{N_{\lambda}-f} \sum_{i=1}^{N_{\lambda}} \frac{\left(\mathcal{F}_{v}^{\text {model }}\left(\lambda_{i}\right)-\mathcal{F}_{v}^{\text {data }}\left(\lambda_{i}\right)\right)^{2}}{\left(\mathcal{F}\left(\lambda_{i}\right)^{2}\right)_{\text {error }}}$ is of the order of unity for most objects. The data with the largest $\chi^{2}$ have the worst signal-to-noise ratios (e.g., SZ 82, VZCha). In Eq. (3), $N_{\lambda}$ is the number of data points, $f$ the degree of freedom, and $\mathcal{F}\left(\lambda_{\mathrm{i}}\right)_{\text {error }}^{2}$ the measurement error of each spectral data point. Following PR03 and KS05 the measurement error is the standard deviation of spectra that was observed on subsequent nights. A root mean square (rms) of $\sim 9 \%$ was determined for TIMMI 2 and $\sim 11 \%$ for LWS observations. However, these errors were derived by also considering parts of the spectra with a significantly lower signal-to-noise ratio, such as the boundaries of the $N$ band and the spectral range where the telluric ozone-band appears. In the following, we assume the rms of $\sim 6 \%$ and $\sim 7 \%$, which we derive from the wavelength interval between $10 \mu \mathrm{m}$ and $12 \mu \mathrm{m}$. For several objects with higher signal-to-noise ratios, we determine the rms by using

$$
\sigma^{2}=\frac{1}{N_{\lambda}-f} \sum_{i=1}^{N_{\lambda}}\left(\mathcal{F}_{v}^{\text {model }}\left(\lambda_{i}\right)-\mathcal{F}_{v}^{\text {data }}\left(\lambda_{i}\right)\right)^{2}
$$

The errors on the fit parameters are calculated using a Monte Carlo method. For every spectrum we generate 100 synthetic spectra, by randomly adding Gaussian noise to the spectrum at each wavelength point. The Gaussian noise has a rms of $\sim 6 \%$ and $\sim 7 \%$, respectively. On each of these spectra, we perform the exact same compositional fit procedure, yielding different values of the fit parameters. From the resulting distribution of all fit parameters, we calculate the arithmetic mean value and standard deviation. 


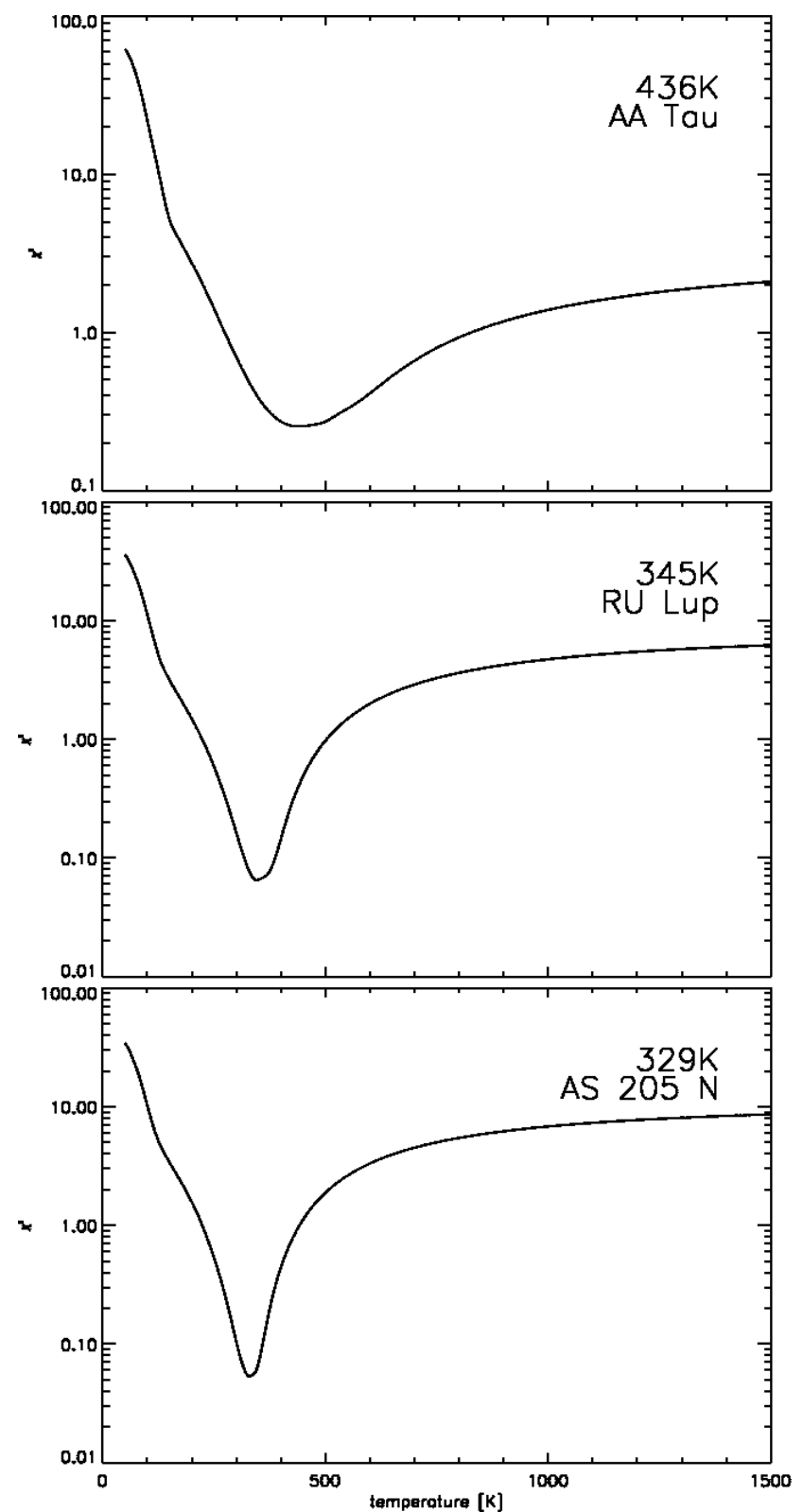

Fig. 2. $\chi^{2}$ of the fits versus the pre-specified temperature $T$. The obtained temperature $T$ for the single fit and the object name are indicated in each plot.

Approximately at $9.7 \mu \mathrm{m}$ a narrow "feature" appears in some spectra (e.g., Haro 1-16) which cannot be fitted by the available emission profiles. We ascribe this artefact to a remnant of data reduction, i.e. sky subtraction, considering the spectral-adjacent, broad atmospheric ozone line at $\sim 9.5 \mu \mathrm{m}$. Additionally, there is a defective readout channel of the TIMMI2 detector between $9.14 \mu \mathrm{m}$ and $9.67 \mu \mathrm{m}$. Because of these two issues, the fitting procedure does not account for the wavelength interval between $\sim 9.2 \mu \mathrm{m}$ and $\sim 9.9 \mu \mathrm{m}$ (Fig. 4). In some spectra (e.g., DQ Tau), a second remnant of sky subtraction appears at $12.7 \mu \mathrm{m}$, where several telluric $\mathrm{OH}$-lines accumulate. In order to improve the fit quality, we went on to cut the spectral data in a narrow wavelength interval at $\sim 12.7 \mu \mathrm{m}$.

Although PAH molecules appear to be abundant throughout the universe (e.g., Lagache et al. 2004; Wu et al. 2005), our spectra can be sufficiently fitted without considering PAHs, although they also show an emission feature near $11.2 \mu \mathrm{m}$ similar

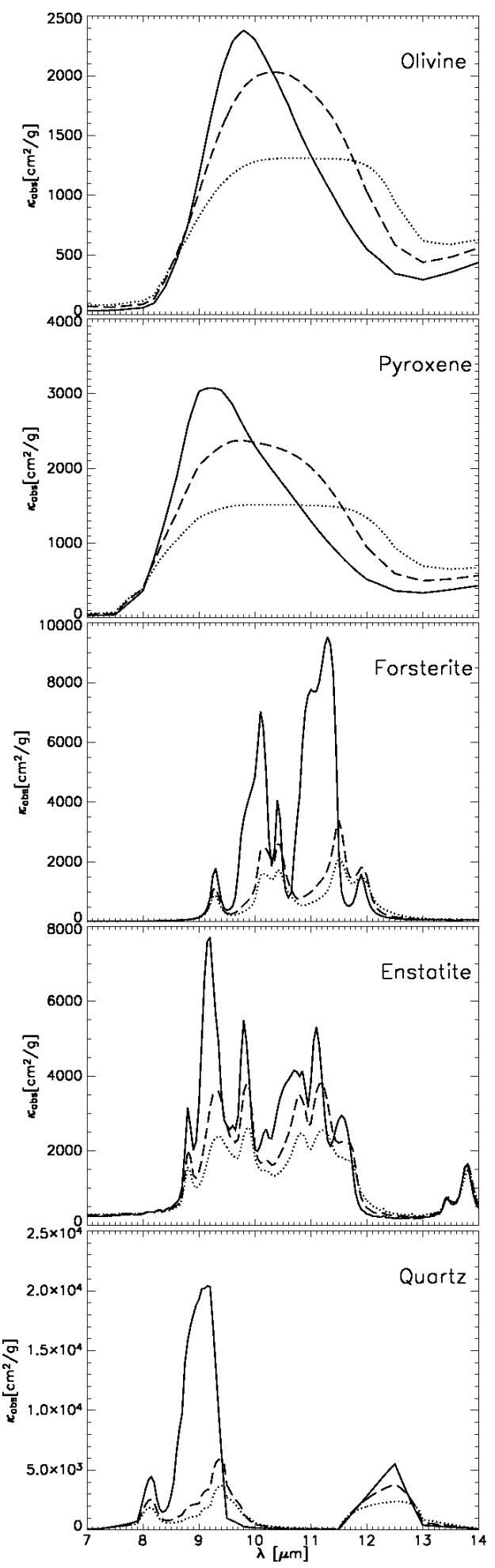

Fig. 3. Opacities of grains with radii $0.1 \mu \mathrm{m}$ (solid lines), $1.5 \mu \mathrm{m}$ (dashed lines), and $2.5 \mu \mathrm{m}$ (dotted lines). With increasing grain size, the maxima shift to longer wavelengths and the emission profile of olivine and pyroxene flattens.

to forsterite. The absence of its $11.3 \mu \mathrm{m}$ feature in the circumstellar disks of (our) T Tauri stars can be explained by the weak stellar UV radiation field compared to the higher-mass HAeBe stars. In fact, PAH molecules are thought to be excited by ultraviolet photons and cooled down by subsequent infrared emission. Furthermore, the circumstellar disk size and disk geometry 


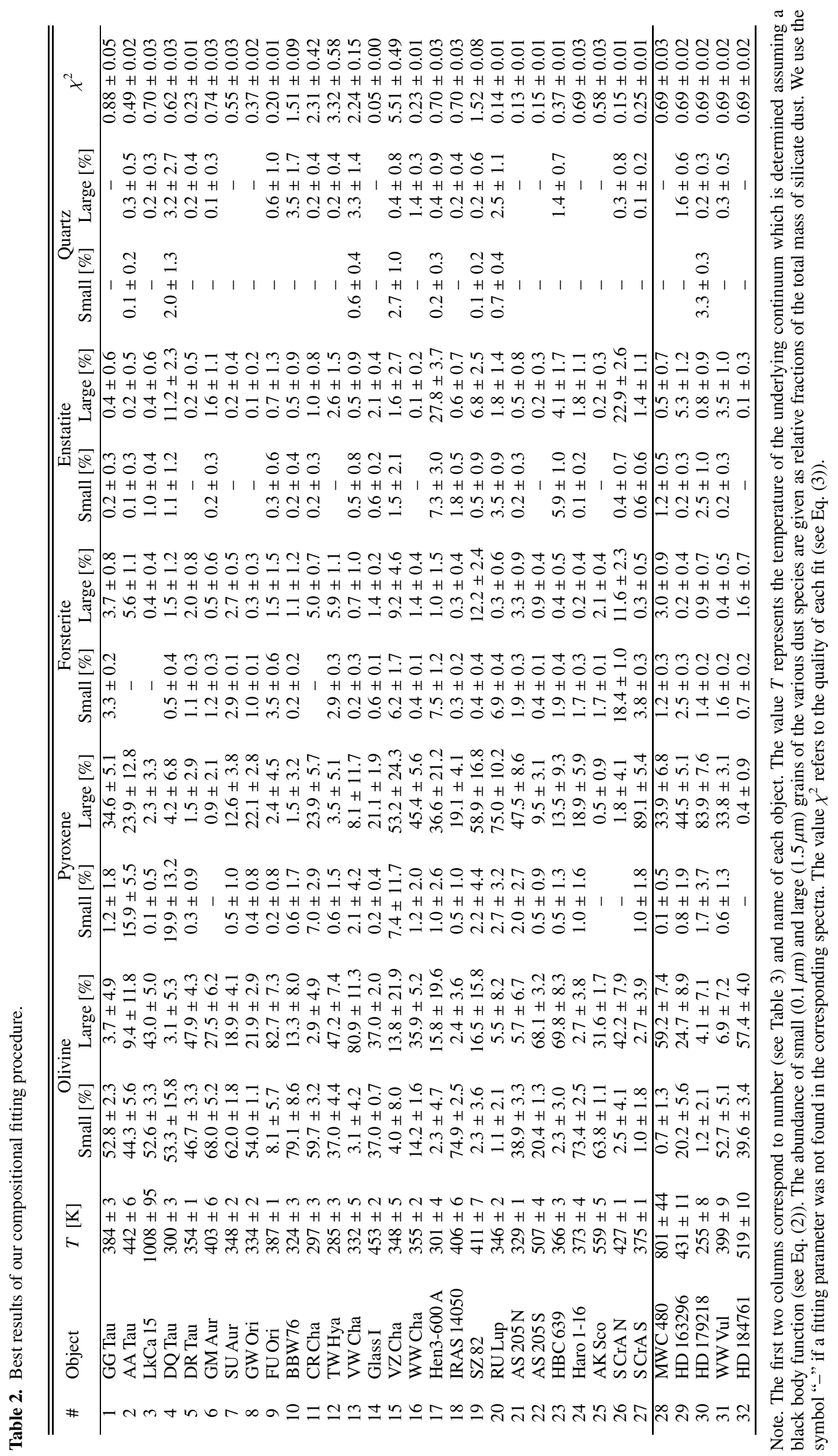



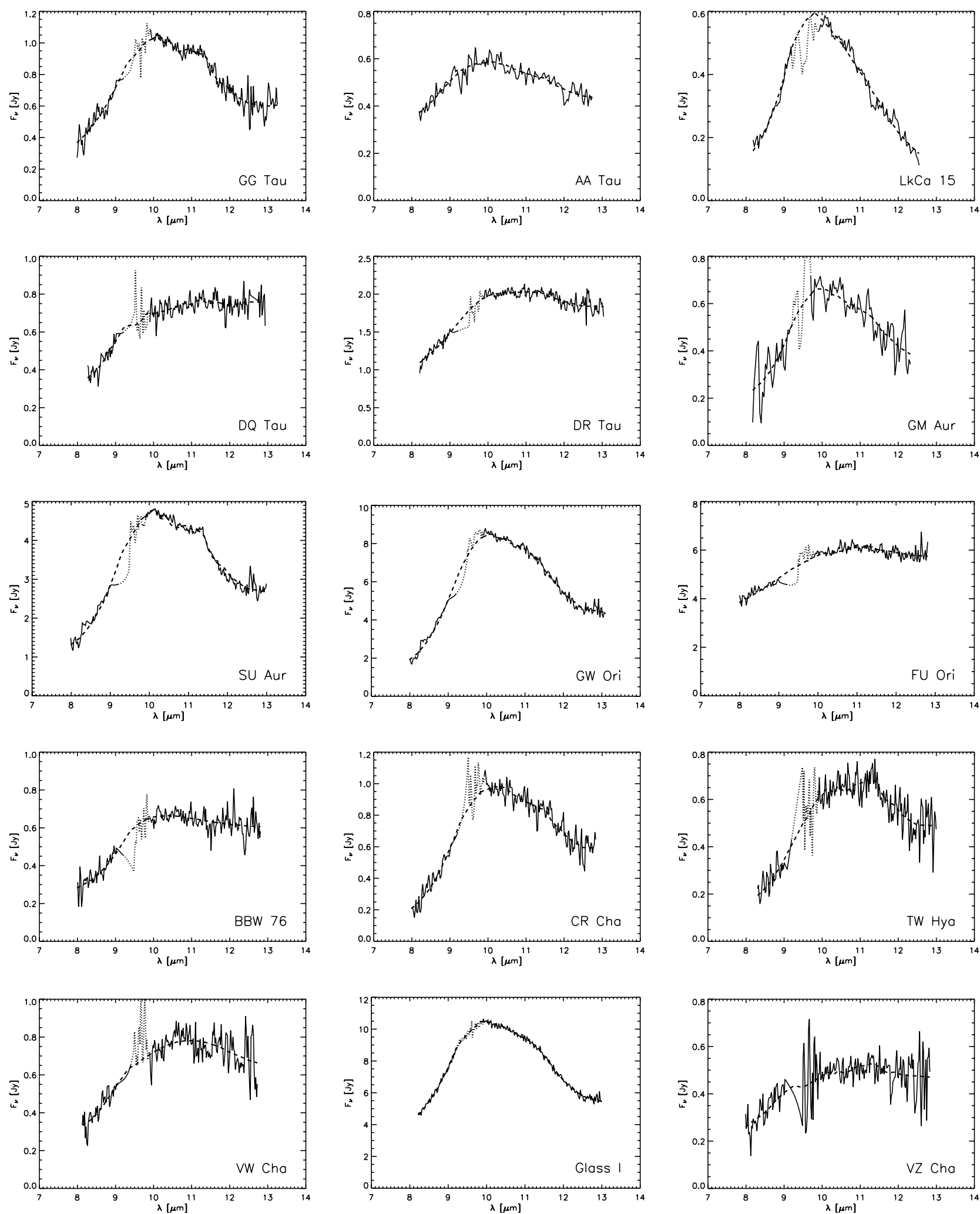

Fig. 4. $N$ band spectra of our sources (solid lines). The best fits to our spectra are also plotted (dashed lines). We cut the wavelength interval $\sim 9.1 \mu \mathrm{m}$ to $\sim 9.7 \mu \mathrm{m}$ from the spectra (dotted line) taking into account the defect readout channel of the TIMMI 2 detector and in order to exclude remnants of the telluric ozone corrections.

affect the presence and strength of the PAH feature as Habart et al. (2004) and Acke \& van den Ancker (2004) show.

Pyroxene is an important part of our dust mixture because it is the dominant component at low temperatures (Gail 2003). BO05 included this dust component when considering its contribution to the short wavelength range of the silicate feature at $\sim 9 \mu \mathrm{m}$ (see Fig. 3). According to our results (see Table 2), the large mass fraction of large pyroxene grains (for example, in VZCha) is suspicious. A corresponding richness of large pyroxene grains was found in HAeBe stars (BO05), too. In 1994, 

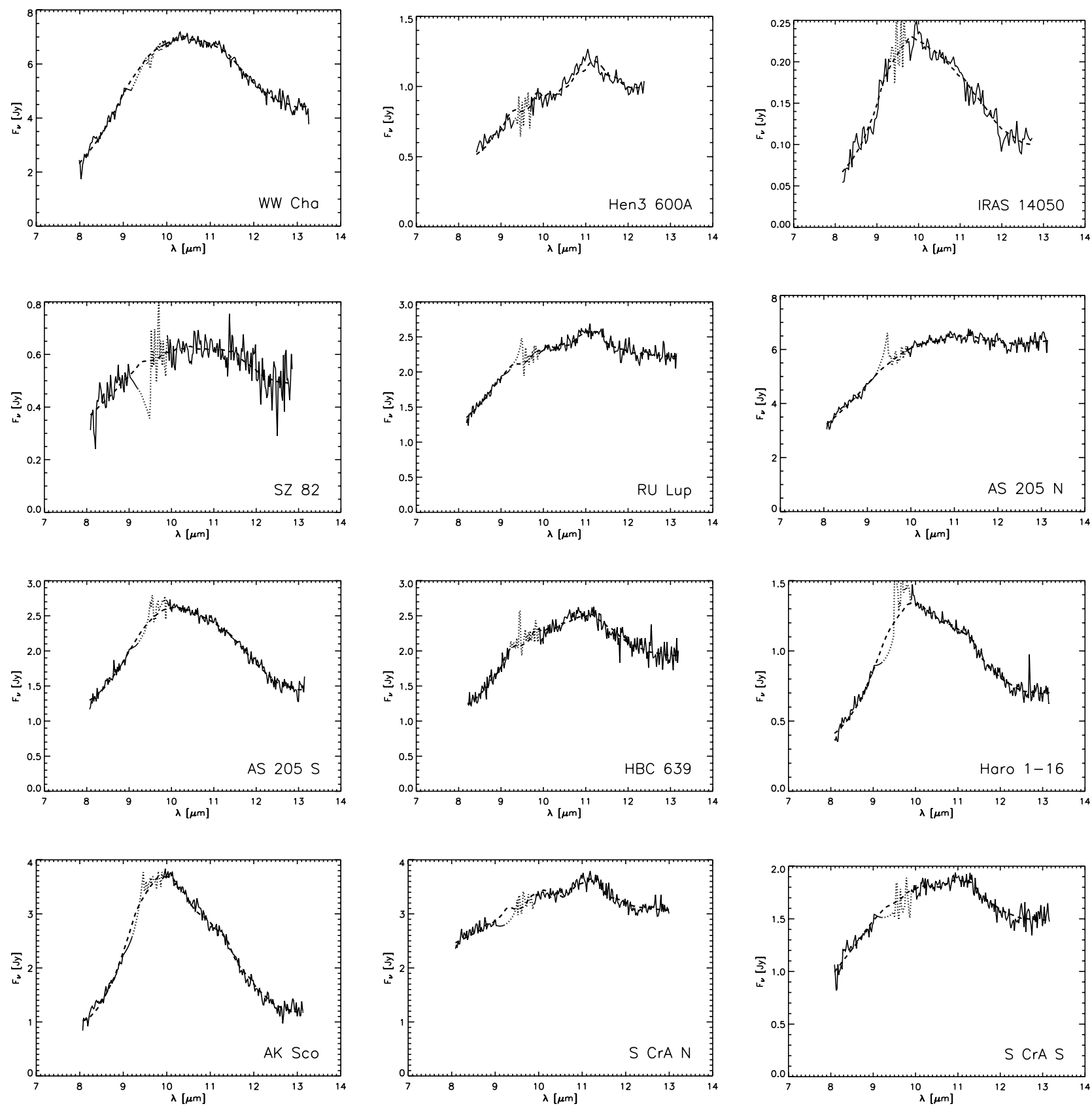

Fig. 4. continued.

Pollack et al. determined that the initial composition of the silicate dust mixture in circumstellar disks only consists of a maximum of $30 \%$ pyroxene dust. Therefore, if the contribution of large pyroxene grains is real, small pyroxene dust grains have already disappeared due to grain growth. If we do not consider pyroxene $^{6}$ in our fitting function, the quantity $\chi^{2}$ increases slightly, about a few percent for the T Tauri stars but up to $100 \%$ for the HAeBe stars HD 163296, HD 179218, and WW Vul.

Apart from the objects Hen 3-600 A and S CrA N for most of the objects, crystalline silicates contribute less than $10 \%$ to the total fit. This result agrees with Pollack et al. (1994), who assumed a crystalline initial contribution of only $10 \%$, while notable amounts of crystalline dust species like enstatite and forsterite are produced in annealing reactions during disk evolution. Gail (2003) shows that crystalline dust dominates in the

${ }^{6}$ Large and small grains. inner parts of protoplanetary disks. However, the temperature is too high in the inner regions of the disk to produce the prominent silicate feature (see Fig. 1). Apparently, the crystallisation of dust is inhibited in the cool circumstellar disks around T Tauri stars, which is confirmed by the minor mass fraction of crystalline dust in contrast to HAeBe stars where a mean crystalline contribution of $\sim 15.0 \%$ was determined (see BO05).

In Table 2, forsterite is the most abundant crystalline dust component in the circumstellar matter of T Tauri systems in 19 of 32 objects. According to Gail (1998) forsterite is the most stable silicate compound thermodynamically, and therefore the final product of the annealing procedure: amorphous grains $\rightarrow$ polycrystalline grains $\rightarrow$ enstatite $\rightarrow$ forsterite. Note that a back-reaction to enstatite is also possible if quartz is present: $\mathrm{Mg}_{2} \mathrm{SiO}_{4}+\mathrm{SiO}_{2} \rightarrow 2 \mathrm{MgSiO}_{3}$ (Rietmeijer et al. 1986). Furthermore, the last step in this cascade can also be inhibited, e.g. by an O- or Mg-deficiency (Fabian et al. 2000) or by 

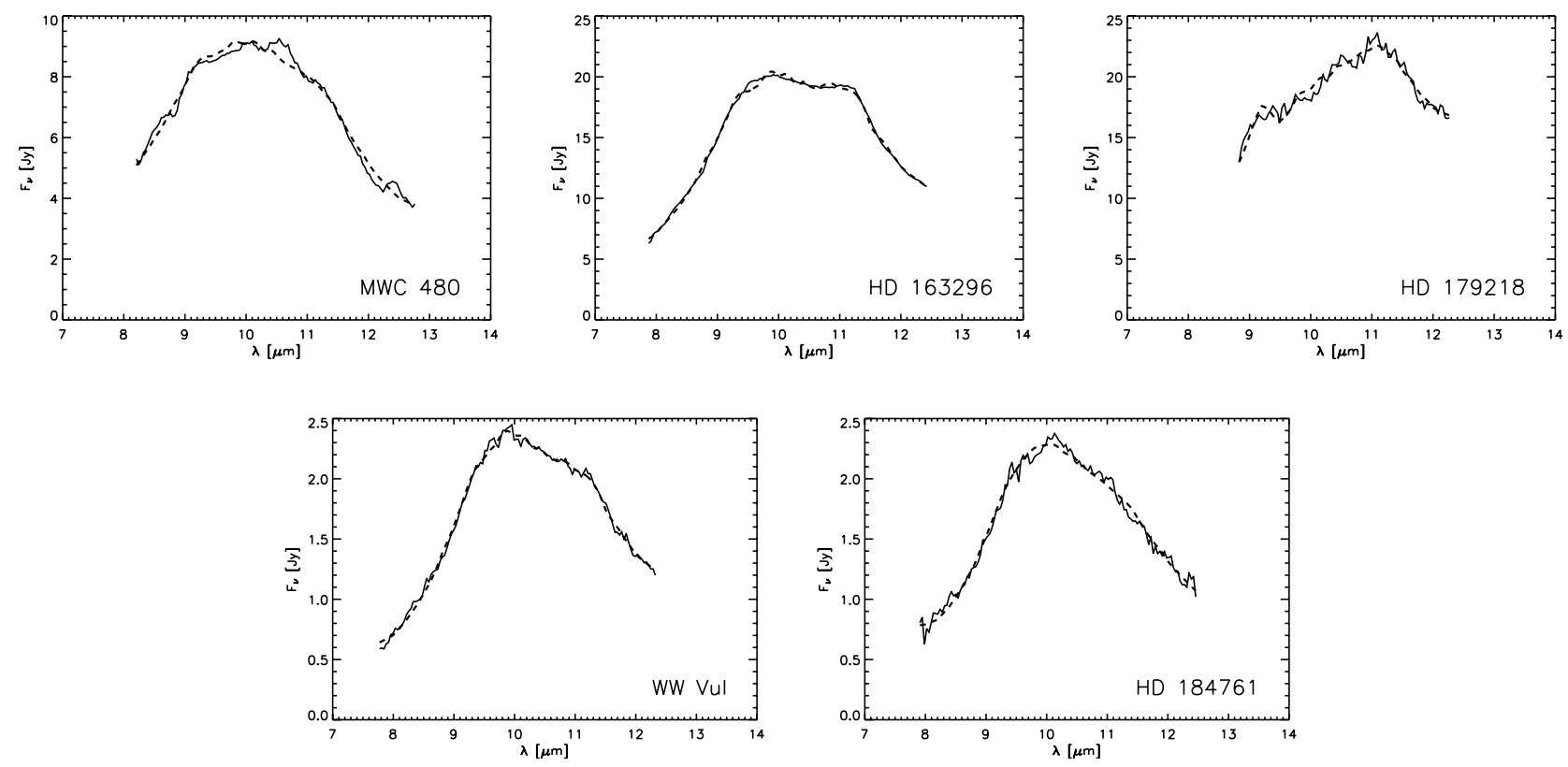

Fig. 4. continued. The spectra of HAeBe stars are shown.

temperatures that are too low (Gail 2003). Thus, the presence of enstatite in circumstellar disks of T Tauri stars cannot be excluded, although it appears less frequently than in HAeBe stars (BO05) ${ }^{7}$.

Quartz is less abundant (see Table 2). This result agrees with the conclusion about the composition of the silicate mixture in stationary protoplanetary disks (see Table 8 in Gail 2003). Gail (2003) determined a fraction of quartz $\leq 5 \%$. Furthermore, the small abundance of quartz - in contrast to the amount in HAeBe stars - could be the result of the quartz emission profile that we implement in our fitting procedure: our profile shows a more discontinous, less smooth curve in contrast to the emission profile that BO05 used.

We conclude by comparing our analysis with previous investigations. Honda et al. (2003) succeeded in fitting the midinfrared spectra of the 10 Myr-old T Tauri star Hen 3-600 A with larger amounts of quartz and less enstatite. The absence of quartz in our fitting results could be the result of "observational selection": the lower resolution of LWS $(R=150)$ in comparison with $R=250$ at the $8.2 \mathrm{~m}$ Subaru Telescope equipped by the Cooled Mid-Infrared Camera and Spectrometer (Honda et al. 2003). But the distinction can also be the consequence of the different fitting functions and the implementation of pyroxene and large crystalline grains in our fitting procedure. It is suspicuous that our spectra tend to rise to longer wavelengths which indicates the negligible contribution of small amorphous grains in contrast to the data of Honda et al. The modified shape of Hen 3-600 A could have its origin in a variable $N$-band emission.

$N$ band spectra of the HAeBe stars HD 179218 and HD 163296 have been previously analysed by BO05, where a higher fraction of large enstatite grains was found for the object HD 179218. This distinction may be due to different spectral resolutions of the instruments (LWS versus TIMMI2) and differences in the fitting procedure. Our fitting results for HD 163296 confirm the results of $\mathrm{BO} 05$.

\footnotetext{
${ }^{7}$ We want to mention that recent mineralogical examinations have shown that enstatite, which could be developed from forsterite, is a final stage of silicate evolution in interplanetary medium and meteorites, in particular. (See Fig. 10; private communication with Dominik Hezel, Institute for Mineralogy, Cologne.)
}

\subsection{Correlations}

While a correlation between feature strength and feature shape has already been observed in HAeBe and T Tauri stars, indicating grain growth (PR03; Acke \& van den Ancker 2004; BO05), we examine the question if there is any correlation between grain growth, crystallinity, and stellar properties like stellar age and luminosity. Here, we include only T Tauri systems.

1. The degree of crystallinity in T Tauri systems correlates with neither stellar age nor luminosity (Figs. 5, 6). It is not clear if the lack of such correlations is a consequence of the uncertainty that adheres to the measurements in Table 1. However, we suppose that a correlation "crystallinity - stellar age" and "crystallinity - stellar luminosity" exists in the inner circumstellar regions, where dust is more exposed to stellar irradiation. Interferometric observations with the MIDI show that the degree of crystallinity increases with decreasing radial distances to the star (van Boekel et al. 2004). But the dust emission from these inner and hotter regions of the circumstellar disk $(>700 \mathrm{~K})$ contributes only marginally to our spectra (Fig. 4) as illustrated in Fig. 1.

With respect to $\mathrm{HAeBe}$ stars, BO05 did not investigate the correlations between crystallinity versus the stellar age and the stellar luminosity. Nevertheless, their analysis of the correlation between the crystalline mass fraction and the stellar mass yielded a weak dependence: objects with larger stellar masses tend to have larger mass fractions of crystalline grains. Assuming a correlation between stellar mass and stellar luminosity, this result disagrees with our study at first glance. But the correlation that was found by BO05 appears only for the more massive HAeBe stars $\left(\gtrsim 3.0 M_{\odot}\right)$. Those HAeBe stars that are less massive are more comparable to T Tauri stars.

2. A clear correlation between the mass fraction of large grains (including large crystalline grains) and stellar luminosity does not exist in our sample of T Tauri stars (Fig. 7). If we exclude objects with the worst signal-to-noise ratio ( $\chi^{2}$, TW Hya, VZCha, and Sz 82), we find that most data points in Fig. 7 are located in the areas limited by the dashed lines. The resulting, potentially existing 


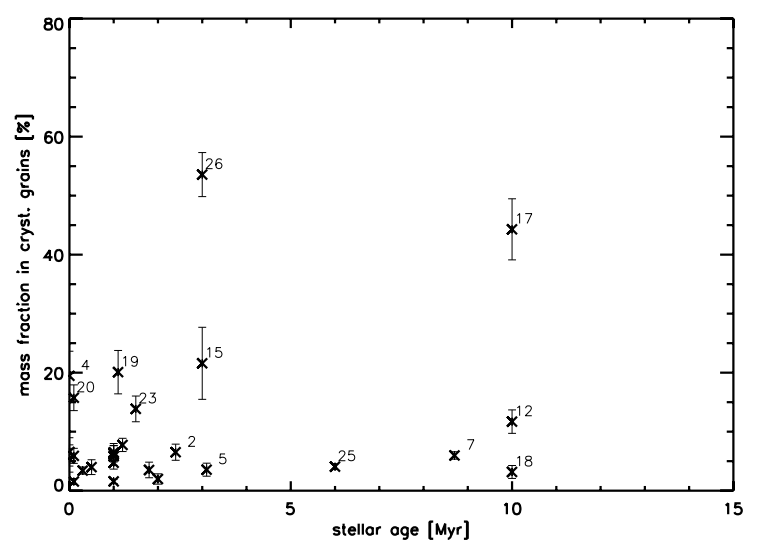

Fig. 5. Mass fraction in crystalline grains vs. stellar age according to Table 2. Numbers of objects are indicated.

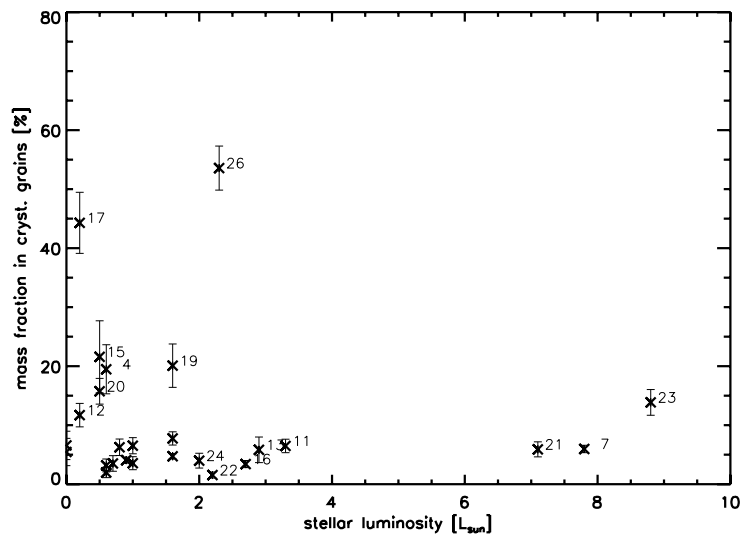

Fig. 6. Mass fraction in crystalline grains vs. stellar luminosity.

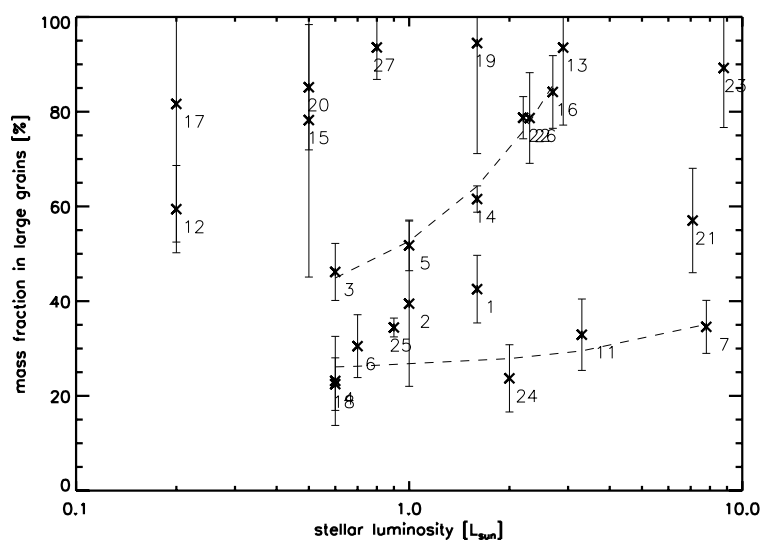

Fig. 7. Mass fraction in large grains versus stellar luminosity. The stellar luminosity is plotted on a logarithmic scale. After excluding objects with the worst signal-to-noise ratio, most objects are located in the area that is limited by the dashed lines. The number of each data point corresponds to the object.

correlation is weak because of the lack of objects with larger and smaller stellar luminosities $\left(>4 L_{\odot}\right.$ and $<0.5 L_{\odot}$ ). BO05 analysed the correlation between stellar mass and mass fraction in large grains. Again, a correlation between both parameters exists only for more massive objects: HAeBe stars with a mass $M \gtrsim 2.5 M_{\odot}$ have a mass fraction of large grains above $85 \%$. Less massive HAeBe stars do not show any dependence on the mass of the central star.

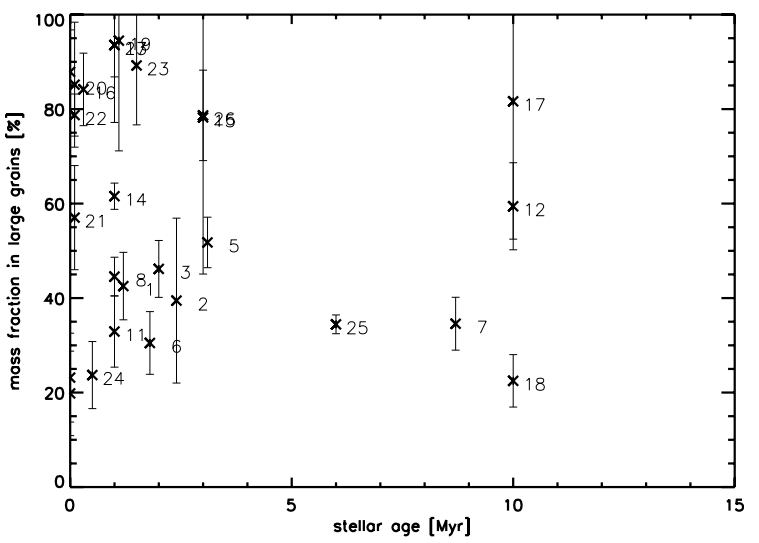

Fig. 8. Mass fraction in large grains vs. stellar age.

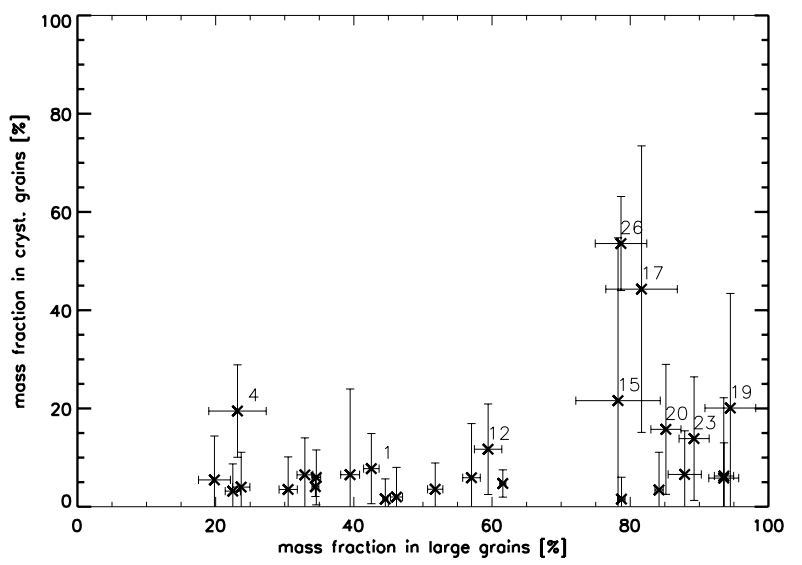

Fig. 9. Relative mass fraction in crystalline grains plotted versus the relative mass fraction in large grains. The number of each data point corresponds to the object in Table 2.

We have to point out that the stellar age does not affect the existence of large grains, which further study shows (see Fig. 8).

3. Figure 9 shows the relative mass fraction in large crystalline grains versus the relative mass fraction in large grains. Apart from the objects Hen 3-600 A, S CrA N, and DQ Tau, most objects show a low-mass fraction of crystalline grains $(<20 \%)$. Such a correlation could also be derived in HAeBe stars (BO05) where crystalline dust was exclusively found in objects with bigger amounts $(>80 \%)$ of large grains. Around half of our T Tauri objects have only a negligible degree of crystallisation in spite of a high mass fraction in large grains. A correlation between grain growth of amorphous grains and grain growth of crystalline grains cannot be found ${ }^{8}$. The existence of such a correlation strongly depends on the rate of crystallisation and grain growth and on the ratio between both quantities. Note also that in some conditions the amorphization of crystalline silicate occurs after ion irradiation (see, e.g., Djouadi et al. 2005).

4. An agreement between T Tauri and HAeBe systems is apparent if the enstatite fraction of crystalline grains is plotted versus the mass fraction of crystalline grains. BO05 found increasing enstatite fractions with increasing degrees of crystallinity. A similar tendency can be found in our sample (see Fig. 10). The explanation for this weak correlation is a

8 Both mass fractions are normalised by the mass fraction of crystalline and the mass fraction of amorphous grains, respectively, considering small and large grains, simultaneously. 


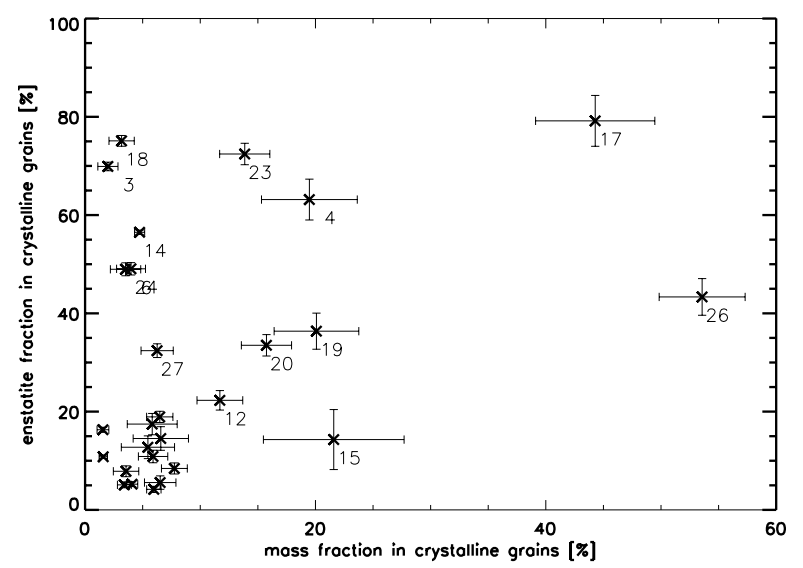

Fig. 10. Enstatite fraction, normalised by the mass fraction of crystalline grains plotted vs. the mass fraction of crystalline grains.

delayed production of forsterite or the reproduction of enstatite from forsterite (see Sect. 4, BO05 and Gail 2003).

Summarising our study, the analysis of the silicate feature of T Tauri systems is complicated by their generally lower brightness in contrast to HAeBe stars (BO05). However, we find correlations which partly agree with the analysis of $\mathrm{BO} 05$, for instance if their correlations are extrapolated to less massive systems.

\section{Composite grains}

The opacities $\kappa_{\mathrm{abs} ; i}(\lambda)$ are conventionally calculated by Mie theory assuming homogeneous dust particles. In reality, collisions with gas and other grains and the interaction with radiation can together lead to grain coagulation, partial grain evaporation, crystallisation, annealing, amorphisation, etc. As a result, composite, partially flaky, and inhomogeneous dust grains evolve with inclusions of vacuum (Dominik et al. 1993; Poppe et al. 2000a,b). The calculations of the optical properties of such grains is a rather difficult problem that can be solved using special algorithms like the discrete dipole approximation (DDA; e.g., Andersen et al. 2002, 2003).

The most frequently used approximation includes considering of homogeneous particles with an average (effective) refractive index derived from effective medium theory (EMT; see Bruggeman 1935; Chýlek et al. 2000). Comparing the DDA and EMT-Mie calculations, Voshchinnikov et al. (2005a) recently discovered that the EMT-Mie approach can give relatively accurate results only for porous particles with "Rayleigh" inclusions $^{9}$. Otherwise, the approach becomes unacceptable when the porosity exceeds $\sim 50 \%$ of the grain volume. At the same time, the optical properties of heterogeneous spherical particles having inclusions of various sizes (Rayleigh and non-Rayleigh) and very large porosity were found to resemble those of spheres with a large number $(\gtrsim 15-20)$ of concentric layers with different compositions.

It was also found that the layered-sphere model predicts a broadening of silicate bands and a shift in the peak position to larger wavelengths as porosity grows, while variations in the feature profile are less significant in the case of the EMT-Mie model (see Voshchinnikov et al. 2005b, for details).

\footnotetext{
9 Inclusions which are small in comparison with the wavelength of incident radiation.
}
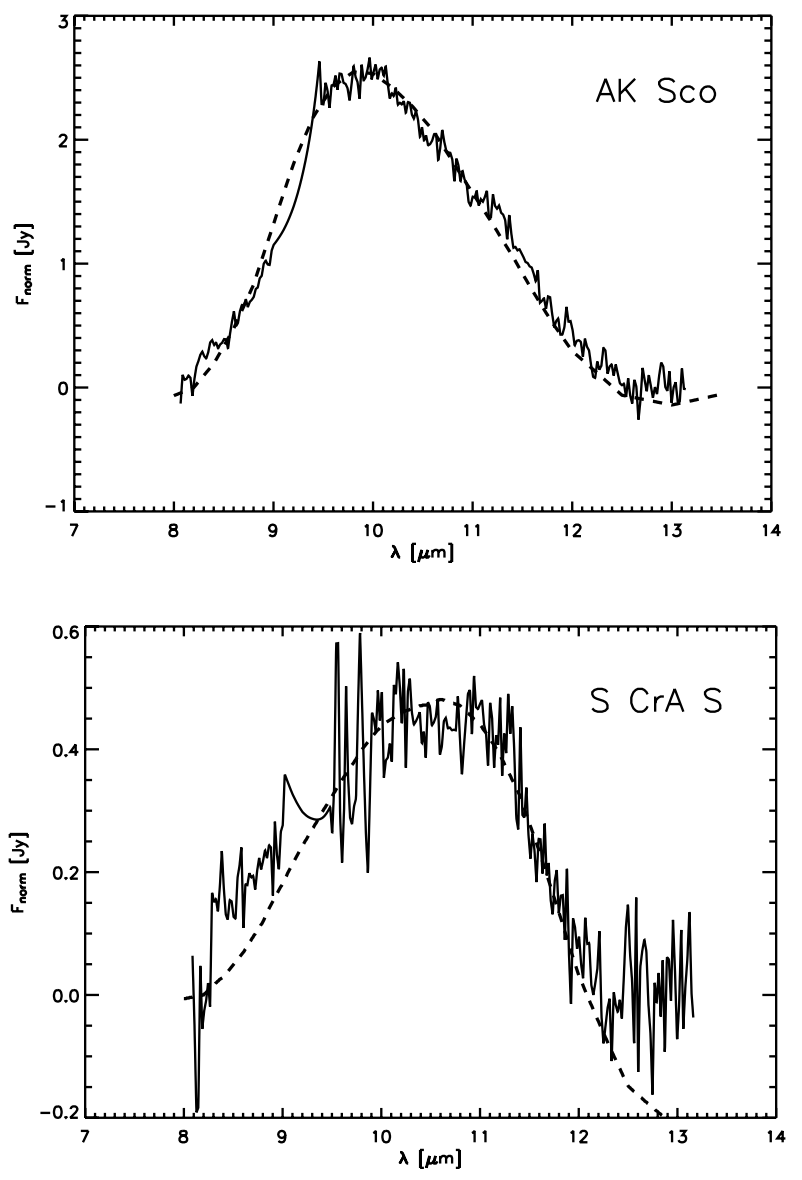

Fig. 11. Normalised, continuum subtracted fluxes of AKSco and S CrA S. The dashed curves represent the results using the multi-layered model for porous grains. The relative volume fraction and the grain radius for AK Sco and S CrA S are presented in Table 3.

Since the cosmic particles can have inclusions of different sizes, here, we apply the model of multi-layered spheres for explanation of the $10 \mu \mathrm{m}$ silicate feature. For simplicity, we consider particles consisting of two materials (carbon and silicate) and 18 concentric layers ${ }^{10}$. Vacuum can be one of the components, too. In contrast to previous modelling with compact grains, the model parameters are grain size, composition, and porosity.

In the context of this layered sphere model, preliminary calculations showed that variations in the volume fractions of vacuum, silicate, and carbon produce diverse profiles of the $10 \mu \mathrm{m}$ feature from strongly peaked to very flat (Voshchinnikov et al. $2005 b)$. It is important to note that different profiles are obtained for small grains with the same compact grain radius $r_{\text {compact }}=0.1 \mu \mathrm{m}^{11}$. This means that the variations in the shape of the silicate band can be related to the change of grain porosity and composition but not necessarily to the grain growth.

As an illustration, Fig. 11 shows the result of our modelling for the objects AK Sco and S CrA S having very different shapes of the silicate band in their spectra. We used glassy olivine, vacuum, and amorphous carbon Be1 (Rouleau \& Martin 1991), along with grains with a compact radius $r_{\text {compact }}=0.1 \mu \mathrm{m}$ in both cases. Crystalline components are not considered. Figure 11 and

\footnotetext{
10 Voshchinnikov et al. (2005a) have shown that this was enough to preclude influence of the order of materials on the results.

11 The quantity $r_{\text {compact }}$ is the radius of the corresponding dust grain without voids.
} 
Table 3. Parameters of composite grains used for modelling the silicate band in the AK Sco and S CrA S spectra (Fig. 11).

\begin{tabular}{cccccc}
\hline \hline Object & $r_{\text {porous }}$ & Olivine & Be1 & Vacuum & $\chi^{2}$ \\
\hline AK Sco & $0.12 \mu \mathrm{m}$ & $49.5 \%$ & $5.5 \%$ & $45 \%$ & 0.57 \\
S CrA S & $0.27 \mu \mathrm{m}$ & $3.35 \%$ & $1.65 \%$ & $95 \%$ & 7.81 \\
\hline
\end{tabular}

Note. The quantity $r_{\text {porous }}$ is the total radius of the porous grains (with vacuum). In Cols. $3-5$ volume fractions of materials are given. The parameter $\chi^{2}$ is explained in Eq. (3).

Table 3 show our best results after varying the relative volume fractions of the three dust components. If the longer wavelength regime $(\lambda \geq 12.3 \mu \mathrm{m})$ of S CrA S is excluded, the fits are satisfying according to Eq. (3). Considering this result and the previous fitting with compact grains, the question certainly arises as to the unambiguous interpretation. More observations should be able to dinstinguish between these two models (see Sect. 6).

There have been previous attempts to compare the silicate feature with the mid-infrared emission behaviour of composite, complex dust grains. Bradley et al. (1999) find that the submicron components of interplanetary dust particles, glass with embedded metals and sulfides (GEMS), show features that are very similar to those in YSOs. The structure of GEMS and the properties of their iron-nickel inclusions refer to their origin. Westphal et al. (2004) suggest, that GEMS were probably formed from crystalline grains by the exposure to hard, ionizing radiation in the stellar outflows from massive stars. Finally, the particles are accelerated in the shock waves of subsequent supernova explosions and distributed over space. The study of the importance of GEMS in circumstellar disks of YSOs and their actual influence on the $10 \mu \mathrm{m}$-silicate-feature is beyond the scope of this study.

\section{Conclusion}

In this study we have investigated the mid-infrared silicate feature of 27 T Tauri and 5 HAeBe stars. The relative mass fractions of amorphous and crystalline, large and small dust grains were derived by fitting a linear combination of the corresponding dust opacities. We assumed that the emission feature has its origin in the optically thin surface layer of the circumstellar disk, while optical thick disk layers only contribute to the underlying continuum of the SED. Because of magnetorotational turbulences and turbulences that arise from Kelvin-Helmholtz instability dust grains with radii $r<1 \mathrm{~cm}$ are assumed to be well mixed vertically in the disk (e.g., Johansen et al. 2005). The relative mass fractions obtained are therefore a copy of the mean composition of micron-sized silicate grains in the whole disk.

A central focus of this study was the question of which physical quantities can be derived from the observation profile of the silicate feature, in general. In terms of an impending violation of the uniqueness of the fitting results, the narrow spectral interval has a very restrictive effect on the number of fitting parameters. Therefore, the number of the selected fitting parameters should be as amsll as possible. Apart from temperature $T$ and a constant term $C_{0}$, we chose the opacities $\kappa_{\mathrm{abs}}$ of following dust species as fitting parameters: $0.1 \mu \mathrm{m}$ - and $1.5 \mu \mathrm{m}$-sized olivine, pyroxene, forsterite, enstatite, and quartz. The PAH emissivities were not implemented. The satisfying fits (see Table 2) underline the negligible abundance of excited PAHs in T Tauri systems, in contrast to HAeBe systems. However, forsterite could overlay the $\mathrm{PAH}$ emission as it has a feature maxima at $11.3 \mu \mathrm{m}$, as well.
As a subsequent result, we found hints that both grain growth and crystallisation appear simultaneously in (our sample of) T Tauri systems, while grain growth is more dominant. This result has to been seen in the context of previous investigations: Mathis et al. (1977) found a particle size distribution of several silicates in interstellar dust that can be reproduced by a power law of a strongly decreasing grain number with increasing grain size. In fact, Bouwman et al. (2001) found negligible mass fractions for large silicate $(\geq 1.5 \mu \mathrm{m})$ grains in interstellar dust as the parent material of dust in circumstellar (HAeBe) disks. However, the conclusion of grain growth is valid only for the case of compact dust grains that consist of one silicate component. Assuming inhomogeneous and flaky dust particles, which can be described by the multi-layered sphere model, for instance, the increase in the porosity of composite grains up to $50 \%$ and higher can be considered as an alternative to grain growth as it generates similar, flat spectra (Voshchinnikov et al. 2005b).

There are indications that all particles larger than $0.1 \mu \mathrm{m}$ are porous. But it is still a central question as to how large the degree of porosity is or if there is even a porosity distribution for grains of certain sizes. Furthermore, the EMT-model and the layered sphere model for porous grains are still competing models that partly provide different results. Although the layered sphere model has its preferences (e.g., both Rayleigh and non-Rayleigh inclusion can be handled), it focuses only on spherical grains. Grains with crystalline inclusion have not been considered, so far.

It is not clear, at all, how strongly porosity affects our fitting results, but according to the layered sphere model, the contribution of large grains would be overestimated as a flat $10 \mu \mathrm{m}$ feature can arise from large grains of any porosity or from very porous small grains, while a narrow feature results from small grains, exclusively.

Considering the fact that $\mathrm{T}$ Tauri systems are less massive than HAeBe stars, our analysis partly agrees with previous results. In fact, our analysis shows that T Tauri systems are a continuation of HAeBe systems at their lower mass end in terms of the properties of the circumstellar dust. Further observations - in particular, of the silicate feature at $20 \mu \mathrm{m}$ (Kessler-Silacci et al. 2006) - and polarization in silicate bands, along with more comprehensive modelling could provide stronger constraints on the existing silicate species in the circumstellar disks of young stars.

Certainly, determination of the individual silicate composition of the disk material is an important result, on its own, but it also has a further consequence for radiative transfer simulations. While radiative transfer simulations of circumstellar disks have been based on the extinction coefficients of an averaged mixture of silicates (e.g., "astronomical silicate", Draine \& Lee 1984), considering of the results of compositional fits such as those presented here are meant to significantly improve disk modelling. Radiative transfer models finally provide further constraints with respect of the fundamental characteristics of the objects like geometry and structure, sky position (inclination), (stellar) luminosity, and object mass (star and disk). Together with the chemical composition of the grains, models of YSOs become quite individual. Furthermore, such complete disk models open up new perspectives, e.g. the "compositional" analysis of absorption features, which are strongly affected by disk structure and inclination.

Acknowledgements. We thank J. Bouwman for discussions and for the opacity data. Furthermore, we thank the referee B. Acke for his helpful suggestions. A. Schegerer and S. Wolf were supported by the German Research Foundation (DFG) through the Emmy-Noether grant WO 857/2-1. 


\section{References}

Adams, F. C., Lada, C. J., \& Shu, F. H. 1987, ApJ, 312, 788

Acke, B., \& van den Ancker, M. E. 2004, A\&A, 426, 151

Akeson, R. L., Walker, C. H., Wood, K., et al. 2005, ApJ, 622, 440

Allen, L. E., Myers, P. C., di Francesco, J., et al. 2002, ApJ, 566, 993

Andersen, A. C., Sotelo, J. A., Pustovit, V. N., \& Niklasson, G. A. 2002, A\&A, 386,296

Andersen, A. C., Sotelo, J. A., Pustovit, V. N., \& Niklasson, G. A. 2003, A\&A, 411,481

Bouwman, J., Meeus, G., de Koter, A., et al. 2001, A\&A, 375, 950

Bradley, J. P., Keller, L. P., Snow, T. P., et al. 1999, Science, 285, 1716

Brooks, D. H., \& Costa, V. M. 2003, MNRAS, 339, 467

Bruggeman, D. A. G. 1935, Ann. Phys., 24, 636

Calvet, N., Muzerolle, J., Briceño, C., et al. 2004, AJ, 128, 1294

Carpenter, J. M., Wolf, S., Schreyer, K., et al. 2005, 129, 1049

Chen, H., Myers, P. C., Ladd, E. F., \& Wood, D. O. S. 1995, ApJ, 443, 625

Chýlek, P., Videen, G., Geldart, D. J. W., et al. 2000, Light Scattering by Nonspherical Particles (San Francisco: Academic Press)

Cotera, A. S., Whitney, B. A., Young, E., et al. 2001, ApJ, 556, 958

Djoudi, Z., d'Hendecourt, L., Leroux, H., et al. 2005, A\&A, 440, 179

Dominik, C., Sedlmayr, E., \& Gail, H.-P. 1993, A\&A, 277, 578

Dorschner, J., Friedmann, C., Gürtler, J., \& Henning, T. 1988, A\&A, 198, 223

Dorschner, J., Begemann, B., Henning, Th., Jäger, C., \& Mutschke, H. 1995, A\&A, 300, 503

Draine, B. T., \& Lee, H. M. 1984, ApJ, 285, 89

Duschl, W., Gail, H.-P., \& Tscharnuter, W. M. 1996, A\&A, 312, 624

Elias, J. H. 1978, ApJ, 224, 453

Fabian, D., Jäger, C., Henning, Th., Dorschner, J., \& Mutschke, H. 2000, A\&A, 364,282

Gail, H.-P. 1998, A\&A, 332, 1099

Gail, H.-P. 2003, in Astromineralogy, ed. Th. Henning (Springer), 55

Greaves, J. S. 2004, MNRAS, 351L, 99

Geoffray, H., \& Monin, J.-L. 2001, A\&A, 369, 239

Gómez, M., \& Mardones, D. 2003, AJ, 125, 2134

Günther, R., Schäfer, \& Kley, W. 2004, A\&A, 423, 559

Habart, E., Natta, A., \& Krügel, E. 2004, A\&A, 427, 179

Haisch, K. E., Lada, E. A., \& Lada, C. J. 2001, ApJ, 553, L153

Hanner, M. S., Brooke, T. Y., \& Tokunaga, A. T. 1995, ApJ, 438, 250

Hartmann, L., Calvet, N., Gullbring, E., \& D'Alessio, P. 1998, ApJ, 495, 385

Hersant, F., Dubrulle, B., \& Huré, J.-M. 2005, A\&A, 429, 531

Honda, M., Kataza, H., Okamoto, Y. K., et al. 2003, A\&A, 585, L59

Jäger, C., Mutschke, H., Dorschner, J., \& Henning, Th. 1998a, A\&A, 332, 291

Jäger, C., Molster, F. J., Dorschner, J., et al. 1998b, A\&A, 339, 904

Johansen, A., Henning, Th., \& Klahr, H. 2005, ApJ, 636, 1121

Krügel, E., \& Walmsley, C. M. 1984, A\&A, 130, 5

Kessler-Silacci, J. E., Hillenbrand, L. A., Blake, A. G., \& Meyer, M. R. 2005, ApJ, 622, 404 (KS05)

Kessler-Silacci, J. E., Augereau, J.-C., Dullemond, C. P., et al. 2006, ApJ, 639, 275

Kitamura, Y., Momose, M., Yokogawa, S., et al. 2002, ApJ, 581, 357

Krist, J. E., Stapelfeldt, K. R., Golimowski, D. A., et al. 2005, AJ, 130, 2778

Lachaume, R., Malbet, F., \& Monin, J.-L. 2003, A\&A, 400, 185

Lagache, G., Dole, H., Puget, J.-L., et al. 2004, ApJ, 154, 112

Lawson, C. L., \& Hanson, R. J. 1974, in Solving Least Squares Problems (Prentice-Hall)

Leinert, C., van Boekel, R., Waters, L. B. F. M., et al. 2004, A\&A, 423, 537
Li, A. 2005, AIPC, 761, 123 [arXiv: astro-ph/0503567]

Manset, N., Bastien, P., \& Bertout, C. 2005, AJ, 129, 480M

Marler, B. W., Jones, B., Pina, R. K., \& Puetter, R. C. 1995, Proc. SPIE, 2475 , 162

Mathieu, R. D., Stassen, K., Basri, G., et al. 1997, AJ, 113, 1841

Mathis, J. S. Rumpl, W. \& Nordsieck, K. H. 1977, ApJ, 217, 425

Meeus, G., Sterzik, M., Bouwman, J., \& Natta, A. 2003, A\&A, 409, 25

Mendoza, E. E. V. 1968, ApJ, 151, 977

Metchev, S. A., Hillenbrand, L. A., \& Meyer, M. R. 2004, ApJ, 600, 435

Molster, F. J., \& Waters, L. B. F. M. 2003, in Astromineralogy, ed. Th. Henning (Springer), 121

Millan-Gabet, R., Schloerb, F. P., \& Traub, W. 2001, ApJ, 2001, 546, 358

Muzerolle, J., Calvet, N., Hartmann, L., \& D’Alessio, P. 2003, 597, 149

Natta, A., Meyer, M. R., \& Beckwith, S. V. W. 2000, ApJ, 534, 838

Natta, A., Prusti, T., Neri, R., et al. 2001, A\&A, 371, 186

Natta, A., Testi, L., Muzerolle, J., et al. 2004, A\&A, 424, 603

Nürnberger, D., Chini, R., \& Zinnecker, H. 1997, A\&A, 324, 1036

Padgett, D. L., Brandner, W., Stapelfeldt, K., et al. 1999, AJ, 117, 1490

Peeters, E., Hony, S., van Kerckhoven, C., et al. 2002, A\&A, 390, 1089

Pollack, J. B., Hollenbach, D., Beckwith, S., et al. 1994, ApJ, 257, 615

Prato, L., Greene, T. P., \& Simon, M. 2003, ApJ, 584, 853

Press, W. H., Teukolsky, S. A., Vetterling, W. T., \& Flannery, B. P. 1986 (Cambridge University Press)

Poppe, T., Blum, J., \& Henning, Th. 2000a, ApJ, 533, 454

Poppe, T., Blum, J., \& Henning, Th. 2000b, ApJ, 533, 472

Przygodda, F., van Boekel, R., Ábráham, P., et al. 2003, A\&A, 412, 43 (PR03)

Przygodda, F. 2004, Ph.D. Thesis, Ruprecht-Karls-Universität, Heidelberg

Reimann, H., Weinert, U., \& Wagner, S. 1998, Proc. SPIE, 3354, 865

Reimann, H., Linz, H., Wagner, R., et al. 2000, Proc. SPIE, 4008, 1132

Rietmeijer, F. J. M., Nuth, J. A., \& Mackinnon, I. D. R. 1986, Icarus, 65, 211

Rouleau, F., \& Martin, P. G. 1991, ApJ, 377, 526

Schütz, O., Meeus, G., \& Sterzik, M. F. 2005, A\&A, 431, 165S

Shu, F. H. 1977, ApJ, 214, 488

Spitzer, W. G., \& Kleinman, D. A. 1960, Phys. Rev., 121, 1324

Stempels, H. C., \& Piskunov, N. 2003, A\&A, 408, 693

Servoin, J. L., \& Piriou, B. 1973, Phys. Stat. Sol. (b), 55, 677

Takami, M., Bailey, J., \& Chrysostomou, A. 2003, A\&A, 397, 675

Tanaka, H., Inaba, S., \& Nakazawa, K. 1996, Icarus, 123, 450

Thi, W. F., van Dishoeck, E. F., Blake, G. A., et al. 2001, ApJ, 561, 1074

van Boekel, R., Waters, L. B. F. M., Dominik, C., et al. 2003, A\&A, 400, L21

van Boekel, R., Min, M., Leinert, Ch., et al. 2004, Nature, 432, 479

van Boekel, R., Min, M., Waters, L. B. F. M., et al. 2005, A\&A, 437, 189 (BO05)

Voshchinnikov, N. V. 2002, in Optics of Cosmic Dust, ed. G. Videen, \& M. Kocifaj (Kluwer), 1

Voshchinnikov, N. V., Il'in, V. B., \& Henning, Th. 2005a, A\&A, 429, 371

Voshchinnikov, N. V., Il'in, V. B., Henning, Th., \& Dubkova, D. N. 2005b, A\&A, 445,167

Weingartner, J. C., \& Draine, B. T. 2001, ApJ, 548, 296

Westphal, A. J., \& Bradley, J. P. 1999, ApJ, 617, 1131

Wolf, S. 2003a, Comput. Phys. Commun., 150, 99

Wolf, S. 2003b, Comput. Phys. Commun., 582, 859

Wolf, S. 2003c, ApJ, 582, 859

Wolf, S., Henning, Th., \& Stecklum, B. 1999, A\&A, 349, 839

Wolf, S., \& Henning, Th. 2000, Comput. Phys. Commun., 132, 166

Wolf, S., Padgett, D. L., \& Stapelfeldt, K. R. 2003, ApJ, 588, 373

Wu, H., Cao, C., Hao, C.-N., et al. 2005, ApJ, 632, 79 